\title{
Short and long-term survival in type $A$ aortic dissection justifies the operative risk and effort
}

\author{
LIN SADI ${ }^{1}$, THEIS TØNNESSEN ${ }^{1,2} \&$ JOHAN PILLGRAM-LARSEN ${ }^{2}$ \\ ${ }^{1}$ Faculty of Medicine and ${ }^{2}$ Department of Cardiothoracic Surgery, Oslo University Hospital Ullevål, \\ University of Oslo, Norway
}

\begin{abstract}
Objectives. To evaluate the survival rate and complications after operation for acute aortic dissection type A. Design. Chart review of all consecutive patients with aortic dissection admitted during 1999-2008 ( $\mathrm{n}=99$ ) to Oslo University Hospital Ullevål, Oslo. Results. Thirty-day mortality was 14 patients and late mortality 21 patients. Twenty-nine patients had no postoperative complications. Cerebral affection was seen in 22 patients. Seventy-nine patients were operated on with deep hypothermic circulatory arrest. Mean circulatory arrest time was 23 minutes (range 12-47). Eighty-three of the patients were cannulated through the femoral artery, with a 30-day mortality rate of $17 \%(\mathrm{n}=14)$ versus $0 \%$ for other cannulations $(n=16)$; and a stroke rate of $24 \%(n=20)$ versus $17 \%(n=2)$ in patients cannulated in the subclavian or axillary artery (no statistically significant difference in either mortality or stroke). Conclusions. Our study confirms that overall mortality and neurological complications are acceptable and the long-term survival rate is good in patients operated on for acute aortic dissection. Circulatory arrest time seems not to affect neurological complications when being relatively short.
\end{abstract}

Key words: aortic dissection, treatment, outcome, survival, complications

\section{Introduction}

Acute aortic dissection type A, of the ascending aorta, is associated with a high rate of mortality and morbidity; and the mandatory treatment is operative to reduce lethal complications. If left untreated, the estimated mortality rate is $50 \%$ during the first 48 hours. The most common causes of death are aortic rupture, stroke, visceral ischemia and cardiac tamponade (1).

The mortality rate and morbidity in type A aortic dissection might vary in different countries depending on transportation distance and duration (e.g. availablility of air ambulance service), and the density of hospitals with cardiothoracic 24-hours service etc. Surgical mortality in operations of type A aortic dissection varies between $15 \%$ and $30 \%$ (2). Recently, the International Registry of Acute Dissection (IRAD) accumulated patients from multiple international sites, and the study showed an overall in-hospital mortality of $25 \%$ (2). Another multicentre study by IRAD examined the long-term survival rate in patients presenting with type A aortic dissection. The contemporary 1-year survival rate is $96 \%$ and 3-year rate is $90 \%$. This study is comparable to our study; the in-hospital complications were dominated by neurological deficits $(22.7 \%, 67$ patients of whom 14 died), myocardial ischemia/infarction $(13.9 \%)$, cardiac tamponade $36 \%$ and acute renal failure (15\%) (3).

The risk stratification system, EuroSCORE, is used in many countries for prediction of cardiac surgical mortality (4). It stands for European System for Cardiac Operative Risk Evaluation, and was developed in 1995 by studying 20000 patients from 128 European countries. Information was collected on 97 risk factors, considering the outcome (survival or death) and the preoperative risk factors. The Logistic EuroSCORE is calculated by using logistic regression methodology. In order to simplify the use of the system and make the risk assessment possible even in the absence of information technology, the simple additive EuroSCORE model was established. If there's a risk factor present in a patient it gives a certain weight, the weights are added to give an approximate percent predicted mortality percentage. In high risk patients, 
the EuroSCORE might underestimate the risk. The logistic EuroSCORE gives a more accurate risk prediction for high risk patients (4).

The aim of the present study was to examine early and long-term mortality in surgical repair of type A aortic dissection, frequency of in-hospital complications and postoperative neurological deficit. Moreover, we wanted to examine whether observed mortality was in the range of predicted mortality by EuroSCORE.

\section{Material and methods}

Between January 1999 and December 2008, 99 consecutive patients underwent repair of type A-aortic dissection at Oslo University Hospital, Ullevål. Patient data and surgical information were registered prospectively into the department's operation database. The final outcome data was retrieved retrospectively from the hospital's data system (PasDoc) and the National Registration Office (Folkeregisteret). All patient's preoperative demographics, comorbid conditions and postoperative complications were analysed. The patients were followed up until Mars 2010; the mean follow-up time being $76 \pm 33$ months, with a follow-up rate of $100 \%$.

Hypertension was defined as blood pressure higher than 140/90 $\mathrm{mmHg}$, renal failure when serum creatinine was higher than $200 \mu \mathrm{mol} / \mathrm{L}$. Patients with postoperative neurological deficit were divided into two groups depending on the clinical presentation. The first group consisted of patients with a moderate sequela, equal hemiparesis. The second group was patients with a major sequela, presenting complications as hemiparesis and dysphasia, aphasia or ischemia in the brain stem.

"Fisher's exact test, two tailed" and "student T test" were used to test for differences; the limit of significance was set to $\mathrm{p}=<0.05$.

\section{Results}

The study group consisted of 99 patients. The mean age at presentation was 60 (range 25-81) years, 23 patients were older than of 70 years. Seventy-eight were males with a mean age of 58 (range 37-81) years; and 21 females with mean age 66 (range 2576) years. Ninety-two of the patients were acutely operated on and seven were electively operated on as defined by EuroSCORE.

The surgical methods were tube graft $(n=86)$; tube graft and reimplantation of the coronary arteries $(n=3)$; tube graft, aortic valve replacement and reimplantation of the coronary arteries (Bentall's procedure, $\mathrm{n}=7$ ) and tube graft with arch surgery $(n=3)$. Seventy-nine of the patients were operated on in deep hypothermic circulatory arrest with a mean arrest time of 23 (range 12-47) minutes; 20 patients (20\%) underwent surgery without deep hypothermic arrest (DHA).

Examining risk factors, we showed that $49 \%$ of the patients had hypertension and $2 \%$ had Marfan's Syndrome. Regarding comorbidity $6 \%$ had chronic obstructive pulmonary disease (COPD), $2 \%$ had a history of earlier myocardial infarction and $4 \%$ of the patients had abdominal aortic aneurysms (Table I).

Thirty-day mortality was 14 patients $(14 \%)$ of whom $5(36 \%)$ were older than 70 years. The overall mortality among patients over the age of 70 was $52 \%$ $(12 / 23)$ and $30 \%(23 / 76)$ for patients younger than 70 years. Thirty-day mortality was $22 \%(5 / 23)$ among patients older than 70 years and $12 \%$ for patients under 70 years of age (Table II).

The 1-year survival rate was $80 \%$ (patients at risk $=86$ ), 3 years rate was $77 \%$ (patients at risk $=72$ ) and 5 years rate was approximately $75 \%$ (patients at risk $=54)($ Figure 1). The cause of early death was stroke in $7(50 \%)$, multi organ failure in $5(36 \%)$, heart failure in $1(7 \%)$ and bleeding in $1(7 \%)$. Late mortality included an additional 21 patients $(21 \%)$ of which $7(33 \%)$ were older than 70 years at the time of operation; 65 patients $(66 \%)$ were still alive by March 2010.

One-third of the patients $(n=29)$ had no postoperative complications. The most common postoperative complications were acute renal failure $(n=23)$, cerebral affection $(n=22)$, respiratory failure $(n=16)$ and reoperation for bleeding $(n=15)$. Severe infections as mediastinitis $(n=3)$ and osteomyelitis of the sternum $(n=1)$ were rare complications. Neurological deficits were seen in 22 patients $(22 \%)$, of whom 20 underwent DHA with a mean circulatory arrest time of $24 \pm 11$ (range 13-47) minutes. The remaining two patients that were operated on without circulatory arrest survived, one with a major sequela and the other with a moderate sequela.

Fifty-nine of the patients (60\%) underwent DHA with no postoperative neurological complications, with mean circulatory arrest time of $22 \pm 7$ minutes.

Table I. Patient characteristics (demographics and comorbid conditions).

\begin{tabular}{lc}
\hline Total number of patients & 99 \\
\hline Age (years, mean \pm SD) & $60 \pm 12$ \\
Female gender & 21 \\
Hypertension & 49 \\
Chronic obstructive pulmonary disease & 6 \\
Abdominal aortic aneurysm & 4 \\
Marfans syndrome & 2 \\
Myocardial infarction & 2 \\
\hline
\end{tabular}


Table II. Mortality rates.

\begin{tabular}{|c|c|c|c|c|c|c|}
\hline & $\begin{array}{l}\text { Thirty-day } \\
\text { mortality (n) }\end{array}$ & $\%$ & $\begin{array}{c}\text { Late } \\
\text { mortality (n) }\end{array}$ & $\%$ & $\begin{array}{c}\text { Overall } \\
\text { mortality (n) }\end{array}$ & $\%$ \\
\hline Patients $\leq 69$ years, $\mathrm{n}=76$ & 9 & $9 / 76=12 \%$ & 14 & $14 / 76=18 \%$ & 23 & $23 / 76=30 \%$ \\
\hline Patients $\geq 70$ years, $n=23$ & 5 & $5 / 23=22 \%$ & 7 & $7 / 23=30 \%$ & 12 & $12 / 23=52 \%$ \\
\hline All patients, $\mathrm{n}=99$ & 14 & $14 / 99=14 \%$ & 21 & $21 / 99=21 \%$ & 35 & $35 / 99=35 \%$ \\
\hline
\end{tabular}

Eight of the patients died because of the neurological complications, the mean circulatory arrest was 25 minutes. The mean circulatory arrest was 27 minutes in patients with a major sequela $(n=2)$, and 22 minutes in patients with a moderate sequela $(n=10)$ (Table III).

Different cannulation techniques for extracorporeal circulation were used, such as femoral $(n=83)$, axillary $(\mathrm{n}=7)$, subclavian $(\mathrm{n}=5)$, aortic $(\mathrm{n}=2)$ and common iliac $(n=1)$ arteries. Eighty-three of the patients were cannulated through the femoral artery, with a 30 -day mortality rate of $17 \%(n=14)$. The 30 -day mortality rate was $0 \%$ in all the other cannulation types. There was a limited number of patients in the different groups, and the difference was not statistically significant ( $\mathrm{p}=0.12)$. Neurological complications were seen in $24 \%(20 / 83)$ of the patients that were cannulated through the femoral artery, $20 \%$ $(1 / 5)$ in subclavian artery cannulation, and $14 \%(1 / 7)$ in axillary artery cannulation. There was no statistically significant difference between the cannulation types and neurological outcome $(\mathrm{p}=0.73)$.

The mean EuroSCORE was 9 (range 6-17) and Logistic EuroSCORE was 19.6 (range 9.0-76.1). In the 30-day mortality group the mean EuroSCORE was 11, and the corresponding Logistic EuroSCORE was 30.7. Patients with a Logistic EuroSCORE less than $10(\mathrm{n}=39)$ presented with a 30-day mortality rate of $5 \%(2 / 39), 15 \%$ (5/34) when EuroSCORE was from 10 to 20 and $27 \%(7 / 26)$ when EuroSCORE was higher than 20 (Table IV).

\section{Discussion}

The in-hospital mortality varies between $15 \%$ and $30 \%$. Recently, IRAD reported an overall in-hospital mortality of $25 \%$. The study included 526 patients from 18 centres in 6 different countries (2). Another Norwegian single centre study shows an in-hospital mortality of $33 \%$ among patients operated for type A aortic dissection (5). Since 30-day mortality is expected to be higher than in-hospital mortality, the $14 \%, 30$-day mortality in our study is lower than the IRAD-reported in-hospital mortality. There might be several reasons for this, e.g. shorter way to the hospital. Our study also shows relatively good long term survival rate. The 1 -year survival rate is $80 \%$, 3 years rate is $77 \%$ and 5 years rate is approximately $75 \%$. This is moderately lower than the reported long-term survival by IRAD, 1-year survival rate of $96 \%$ and 3 years survival rate of $90 \%$. The survival analysis in this study started on the day of discharge and, thereby, excluding the in-hospital mortality. Previous studies have reported a 1-year survival rate between $52 \%$ and $94 \%$, and a 5-year survival rate between $45 \%$ and $88 \%$ (3). Thus, overall mortality in our study is acceptably low.

The use of deep hypothermic circulatory arrest $\left(18^{\circ} \mathrm{C}\right)$ is most commonly applied in the operation for type A aortic dissection. This procedure is believed not to provide adequate protection of the brain for longer than 30 minutes. The safe period of circulatory arrest time can supposedly be increased up to

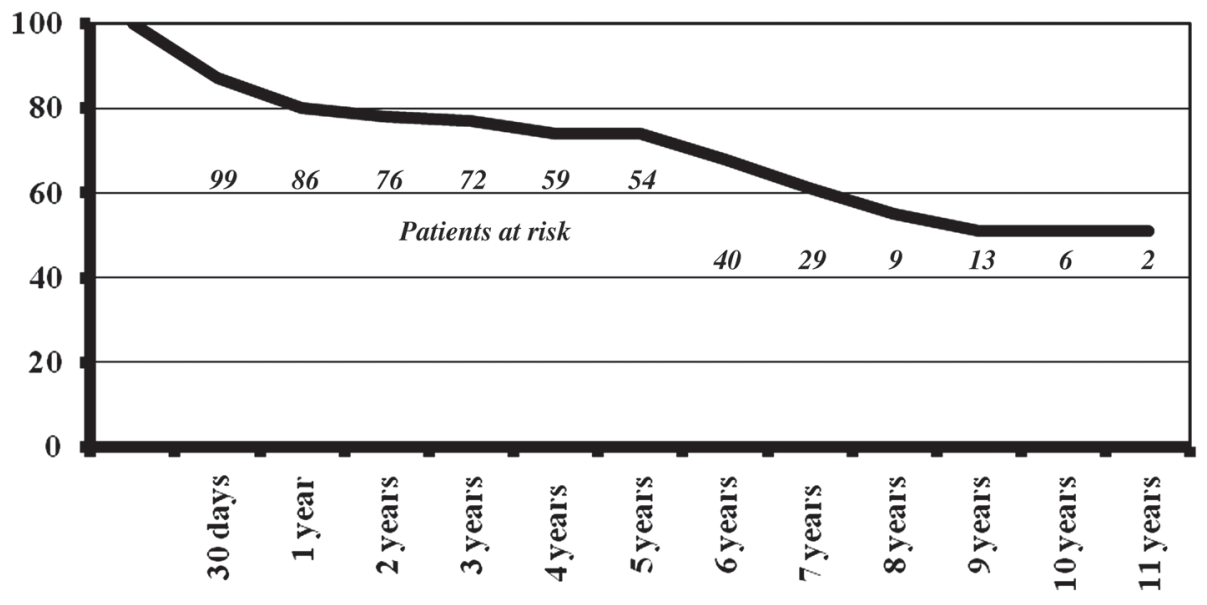

Figure 1. Long term survival curve. 
Table III. Post-operative neurological complications and circulatory arrest time.

\begin{tabular}{lccc}
\hline $\begin{array}{l}\text { Neurological } \\
\text { complications }\end{array}$ & Number & Percentage & $\begin{array}{c}\text { Circulatory arrest } \\
\text { time (minutes) }\end{array}$ \\
\hline Totally & 20 & $100 \%$ & 24 \\
Mortality & 8 & $40 \%$ & 25 \\
Major sequela & 2 & $10 \%$ & 27 \\
Moderate sequela & 10 & $50 \%$ & 22 \\
\hline
\end{tabular}

60 minutes or more with antegrade selective cerebral perfusion (ASCP) (6). One study shows a correlation between the circulatory arrest time and cerebral injury where the incidence of neurological dysfunction increased rapidly beyond 50 minutes of arrest time (7). Another study reported that the safe period before strokes develop is approximately 40 minutes (8). The circulatory arrest time seems not to affect the postoperative neurological complications in our study. This is most likely explained by the relatively short arrest times, where the arrest time exceeded 40 minutes in only three patients.

In $18-30 \%$ of the cases (9), type A aortic dissection is accompanied by neurological symptoms. The symptoms are caused by either bad hemodynamic state of the patients or by malperfusion of the arteries of the brain or spinal cord. Hypoperfusion of the brain can cause either transitory or permanent clinical manifestations, like hemiparesis, hemiplegia or deep coma. As a result of hypoperfusion of the spinal cord, paraparesis occurs in $2-5 \%$ of the treated patients. One study shows a significant difference in neurological complications in patients operated with antegrade $(7.5 \%)$ versus retrograde $(12.5 \%)$ perfusion (9). The frequency of postoperative neurological complications in our study was acceptably low.

Most of our patients were cannulated through the femoral artery. This procedure might, however, be associated with complications as propagation of a retrograde dissection, embolization of lumen debris and malperfusion that might lead to ischemia in end organs and cerebral injury (6). In the past years also other cannulation sites have been chosen in our department, but we do not have enough patients in our cohort to compare the methods and draw any conclusions on which site is the better. With relatively few procedures per surgeon per year, we think that a fast, standardized procedure as cannulation through the femoral artery is acceptable, although modifications must be carried out when required. This procedure might, however, be associated with complications as propagation of a retrograde dissection, embolization of lumen debris and malperfusion which might lead to ischemia in end organs and cerebral injury. Antegrade cerebral perfusion is reported to be safer and might reduce perfusion related morbidity, hospital mortality and stroke rate compared to femoral cannulation (9). This can be
Table IV. Logistic EuroSCORE versus 30-day mortality.

\begin{tabular}{lc}
\hline Logistic EuroSCORE & Thirty-days mortality \\
\hline$<10$ & $2 / 39(5 \%)$ \\
$10-20$ & $5 / 34(15 \%)$ \\
$>20$ & $7 / 26(27 \%)$ \\
\hline
\end{tabular}

The logistic EuroSCORE reflects the mortality, Fisher's exact test, onetailed gives $p=0.004$ for a versus $c ; p=0.16$ for a versus $b$; $\mathrm{p}=0.19$ for $\mathrm{b}$ versus $\mathrm{c}$.

achieved either by direct cannulation into one or both carotid arteries or by cannulation of the axillary artery (Table V). The axillary artery is rarely affected by extensive atherosclerotic lesions, which is commonly found in the femoral artery $(6,10,11)$, it also facilitates perfusion to the carotid artery and vertebral artery. If only the axillary artery is cannulated, adequate cerebral perfusion is dependent on the status of the circle of Willis. A recent anatomic study by Merkkola et al. (12) confirms large anatomic varations of the circle of Willis, where the circulation to the left hemisphere would have been insufficient in 14-17\%. However, the result indicates that in most patients undergoing aortic arch operation, the circulation of the left hemisphere would be sufficient. Due to the small risk of insufficient circulation, the authors recommend that cerebral protection deep hypothermia should always be included; and where possible, do a preoperative examination of the cerebral arteries by computed tomography angiogram to identify high-risk patients. In another anatomic study, Manninen et al. (13) present data concerning the adequacy of cerebral circulation after stent graft exclusion of the left subclavian artery. The study shows that due to anatomic variations, unprotected left subclavian artery occlusion might lead to insufficient posterior cerebral circulation. Of the studied individuals, 5.4\% were at risk for acute neurological complications after unprotected closure of the left subclavian artery. This necessitates diagnostic imaging (when suitable) of the posterior and anterior intracranial circulation. A preoperative diagnostic imaging of the cerebral circulation will, however, not be suitable in patients with type A aortic dissection that are hemodynamically unstable. Another helpful tool can be the noninvasive oxygen saturation

Table V. Mortality rate in the different cannulation methods used.

\begin{tabular}{lcccc}
\hline Cannulation & Alive & $\begin{array}{c}\text { Thirty-days } \\
\text { morality }\end{array}$ & $\begin{array}{c}\text { Late } \\
\text { mortality }\end{array}$ & Total \\
\hline Femoral artery & $50(60 \%)$ & $14(17 \%)$ & $19(23 \%)$ & 83 \\
Subclavian artery & $4(80 \%)$ & 0 & $1(20 \%)$ & 5 \\
Axillary artery & $6(86 \%)$ & 0 & $1(14 \%)$ & 7 \\
Common iliac artery & $1(100 \%)$ & 0 & 0 & 1 \\
Aorta & $2(100 \%)$ & 0 & 0 & 2 \\
Unknown & 1 & 0 & 0 & 1 \\
Total & 64 & 14 & 21 & \\
\hline
\end{tabular}




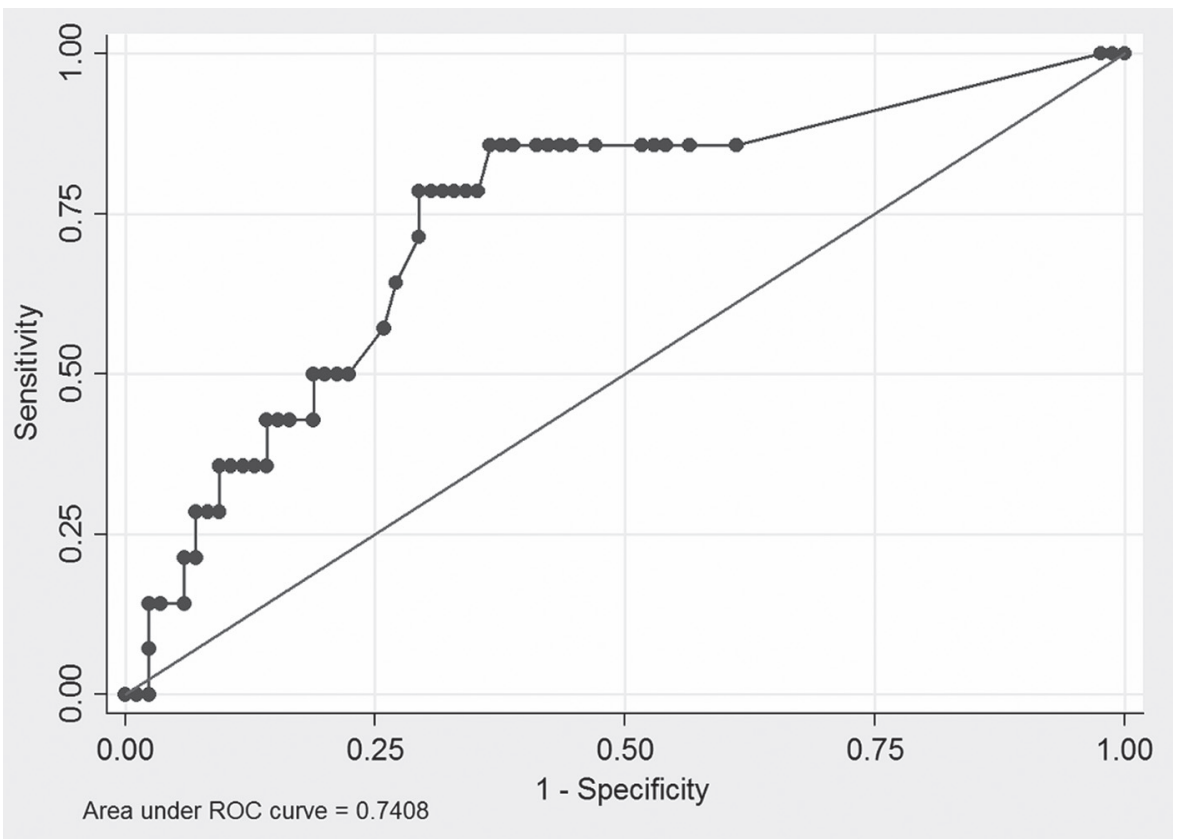

Figure 2. Logistic EuroSCORE versus early mortality: Text: ROC curve shows area under curve $=0.741$ with $95 \%$ confidence interval (0.643-0.825), $\mathrm{p}=0.003$.

system placed on the patients forehead like the INVOS near infrared light cerebral oxymetry system (14) guiding the surgeon when perfusion of the left side of the brain is insufficient, and further steps have to be undertaken. This technique makes is easier to control an adequate cannula positioning and reduce the cerebral complications due to cerebral malperfusion.

Some independent predictors of follow-up mortality have been reported by IRAD, the predictors were age (over 70 years), gender (female), history of atherosclerosis and history of previous cardiac surgery (3). IRAD has also studied preoperative predictors of mortality, such as history of aortic valve replacement, hypotension as a symptom of type A dissection, limb ischemia, migrating chest pain, tamponade or shock (2). All these factors are important determinants of stroke and mortality. We did not examine these factors in our study, but we used the EuroSCORE to predict operative mortality.

The preoperative risk stratification tool, Logistic EuroSCORE, reflects the mortality rate in our selected material and gives a good prediction of early mortality (Figure 2). Although EuroSCORE and Logistic EuroSCORE have been reported to overestimate mortality-risk in other patient groups such as coronary artery bypass surgery (15).

\section{Conclusion}

With a 30 -day mortality of $14 \%$ and a 5 -year survival rate of $75 \%$ (patients at risk $=54$ ), our study shows that the operation for acute aortic dissection, type A in a low volume cardic surgical unit has an acceptable risk. Neurological deficits were seen in 22 out of $99(22 \%)$ patients of whom eight died. We could not document that circulatory arrest time or cannulation site influenced neurological complications, a finding that might be linked to the relatively small number of patients in each group and the relatively short circulatory arrest times.

\section{Acknowledgements}

We are indebted to senior researcher Michael Abdelnoor, Ph.D., Department of Biostatistics and Epidemiology, Oslo University Hospital, for his statistical assistance. The study was approved by the hospital's ombudsman for patients, interests and safety.

Declaration of interest: The authors report no conflicts of interest. The authors alone are responsible for the content and writing of the paper.

\section{References}

1. Knipp BS, Deeb GM, Prager RL, William CY, Upchurch GR Jr., Patel HJ. A contemporary analysis of outcomes for operative repair of type A aortic dissection in the United States. Surgery. 2007;142:524-8.

2. Trimarchi S, Nienaber CA, Rampoldi V, Myrmel T, Suzuki $\mathrm{T}$, Metha $\mathrm{RH}$, et al. Contemporary results of surgery in acute type A aortic dissection: The International Registry of Acute Aortic Dissection experience. J Thorac Cardiovasc Surg. 2005;129:112. 
3. Tsai TT, Evangelista A, Nienaber CA, Trimarchi S, Sechtem U, Fattori R, et al. Long-term survival in patients presenting with type A acute aortic dissection: Insights from the International Registry of Acute Aortic Dissection (IRAD). Circulation. 2006;114:1350-6.

4. Roques F, Nashef SA, Michel P, Gauducheau E, de Vincentiis C, Baudet E, et al. Risk factors and outcome in European cardiac surgery: Analysis of the EuroSCORE multinational database of 19030 patients. Eur J Cardiothorac Surg. 1999;15:816-22.

5. Bartnes K, Christensen T, Myrmel T. Follow-up after acute aortic dissections-time to differentiate? Scand Cardiovasc J. 2011;45:48-53.

6. Trimarchi S, Myrmel T, Panza A, Benedetto DG, Rampoldi V. Vascular surgical options. In: Baliga RR, Nienaber CA, Isselbacher EM, Eagle KA, eds. Aortic dissection and related syndromes. New York: Springer Science + Business media, LLC; 2007. pp. 334-46.

7. Ergin MA, Griepp EB, Lansman SL, Galla JD, Levy M, Griepp RB. Hypothermic circulatory arrest and other methods of cerebral protection during operations on the thoracic aorta. J Card Surg. 1994;9:525-37.

8. Svensson LG, Crawford ES, Hess KR, Costelli JS, Raskin S, Shenaq SA, et al. Deep hypothermia with circulatory arrest. Determinants of stroke and early mortality in 656 patients. J Thorac Cardiovasc Surg. 1993;106:19-28.
9. Karabdic IH, Pasic M, Omerbasic E. Preoperative and postoperative neurological complications in aortic dissection type A. Med Arh. 2010;64(1):15-16.

10. Svensson LG, Blackstone EH, Rajeswaran J, Josepf FS, Lytle BW, Stawinski GG, et al. Does the arterial cannulation site for circulatory arrest influence stroke risk? Ann Thorac Surg. 2004;78:1274-84.

11. Eusanio DM, Schepens MA, Morshuis WJ, Dossche KM, Bartolomeo DR, Pacini D, et al. Brain protection using antegrade selective cerebral perfusion: A multicenter study. Ann Thorac Surg. 2003;76:1181-8.

12. Merkkola P, Tulla H, Ronkainen A, Soppi V, Oksala A, Koivisto $\mathrm{T}$, et al. Incomplete circle of willis and right axillary artery perfusion. Ann Thorac Surg. 2006;82:74-9.

13. Manninen H, Tulla H, Vanninen R, Ronkainen A. Endangered cerebral blood supply after closure of left subclavian artery: Postmortem and clinical imaging studies. Ann Thorac Surg. 2008;85:120-6.

14. Rubio A, Hakami L, Munch F, Tandler R, Hariq F, Weyand M. Noninvasive control of adequate cerebral oxygenation during low-flow antegrade selective cerebral perfusion on adults and infants in the aortic arch surgery. J Card Surg. 2008 ;(5):474-9.

15. Engebretsen KV, Friis C, Sandvik L, Tønnessen T. Survival after CABG-better than predicted by EuroSCORE and equal to general population. Scand Cardiovasc J. 2009;43:123-8. 